\title{
Organic distributed feedback laser biosensor
}

\author{
A-M Haughey*, G A Burley ${ }^{\dagger}$, A L Kanibolotsky ${ }^{\dagger}$, P J Skabara ${ }^{\dagger}$, M D Dawson* and N Laurand* \\ *Institute of Photonics, SUPA, University of Strathclyde, Glasgow G4 0NW, UK \\ ${ }^{\dagger}$ WestCHEM, Department of Pure and Applied Chemistry, University of Strathclyde, Glasgow G1 1XL, UK \\ Email: annemarie.haughey@strath.ac.uk
}

\begin{abstract}
We present a truxene-based distributed feedback laser sensor and demonstrate its sensing capabilities. Results for bulk refractive index sensing, detection of nano-layer adsorption at the laser surface and specific biomolecule sensing are shown.
\end{abstract}

\section{INTRODUCTION}

Evanescent sensors are label-free sensors that measure a change in the refractive index of the medium at the sensor surface. Such sensors are ideal for biosensing applications due to their capacity for label-free detection, multiplexing and their incorporation into lab-on-chip systems and they have been shown to provide good sensitivity and specificity for biosensing [1]-4]. Distributed feedback (DFB) lasers represent one type of evanescent biosensor and have several attractive attributes such as simple fabrication and implementation, high sensitivity and compactness. The surface of a DFB laser can be functionalized for the detection of specific analytes; binding of target analytes produces a change in refractive index at the laser surface which translates to a corresponding change in emission wavelength that is detected. Existing DFB laser biosensors utilize a dye-doped polymer as the gain material and have been shown to detect a variety of different biomolecules [5], 6]. Here, we introduce an 'allorganic' truxene oligofluorine based DFB laser biosensor. By using an organic semiconductor as the gain material, which is less prone to quenching than dye-doped polymers, there is potential to enhance laser, and therefore sensing, characteristics.

We present our sensor's current capabilities for bulk refractive index sensing via measuring the response to a range of different glycerol solutions and a comparison of our experimental results to simulated results from an analytical model. Detection of layer-by-layer adsorption of polyelectrolytes to the laser surface is demonstrated and initial results for specific biomolecule (avidin) detection are also included.

\section{DeVice CONCEPT AND FABRICATION}

The sensor is based on a second-order verticallyemitting DFB laser and consists of a three-layer planar waveguide with a thin gain layer of thickness $t$. The substrate, optical epoxy (Norland 65), is patterned with a periodic nanostructure (period, $\Lambda, 276 \mathrm{~nm}$; grating depth, $\delta \mathrm{t}, 50 \mathrm{~nm}$ ) which provides both the out-coupling and feedback mechanism for the laser, as shown in figure 1 . The gain layer is an organic semiconductor composed of fluorescent tris(terfluorenyl)truxene (T3). T3 has a refractive index of 1.81 at $430 \mathrm{~nm}$ in a thin film [7] which is higher than the refractive indices of the substrate and superstrate, i.e. forming a planar waveguide.

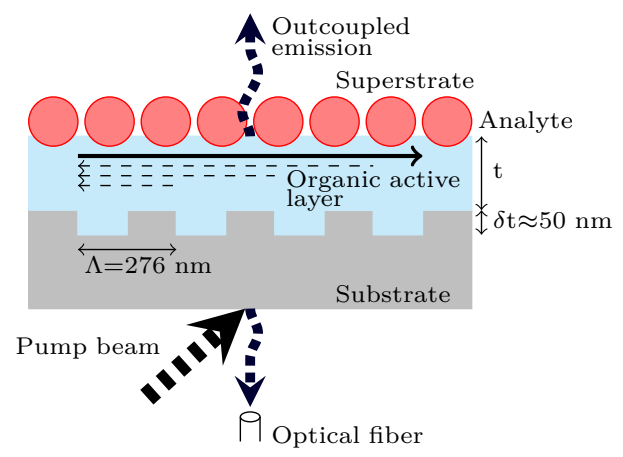

Figure 1. Schematic of the DFB laser structure.

Emission from the DFB laser is governed by the Bragg equation, $\lambda=n_{\text {eff }} \Lambda$, where $\lambda$ is the Bragg wavelength, $\mathrm{n}_{\text {eff } f}$ is the effective refractive index of the mode, and $\Lambda$ is the grating period as defined previously. Changes in $n_{e f f}$ result in a change in emission wavelength from the sensor. All values used to determine $\mathrm{n}_{e f f}$, excluding superstrate refractive index, are fixed, therefore changes in the optical properties at the sensor surface, such as the presence of an analyte, can be detected by monitoring the shift in the emission wavelength of the laser.

The sensor was fabricated by drop-coating an optical epoxy onto a master grating, with a piece of acetate sheet on top, and photocuring with a UV lamp for $50 \mathrm{~s}$. After curing, the grating imprinted epoxy was peeled from the master grating and cured for a further 60 minutes. T3 solution $(20 \mathrm{mg} / \mathrm{mL})$ was prepared in toluene and $20 \mu \mathrm{L}$ spin-coated onto the grating imprinted epoxy at $3.2 \mathrm{krpm}$ for $90 \mathrm{~s}$. Sensors were pumped through the substrate, at an external angle of $\approx 45^{\circ}$, with a frequency-tripled, $\mathrm{Q}$ switched Nd:YAG laser $(355 \mathrm{~nm}, 10 \mathrm{~Hz}$ repetition rate) and out-coupled emission was collected by an optical fiber and transferred to a grating-coupled CCD spectrometer, as shown in figure 1

\section{Results}

\section{A. Bulk refractive index sensing}

Bulk refractive index sensing was demonstrated by immersing the sensor in glycerol-water solutions of different volume ratios $(5 \%-60 \%)$, hence different refractive indices. The laser emission wavelength was found to redshift $>5 \mathrm{~nm}$ for increasing glycerol solution refractive index corresponding to a refractive index detection sensitivity 
of $21 \mathrm{~nm} /$ refractive index unit, shown in figure 2 insert. The results are compared to, and shown to agree with, a theoretical model, shown in 2. Correlation between experimental and modeled data indicates that the model can be used to infer structural changes to optimize the sensor structure for sensing.

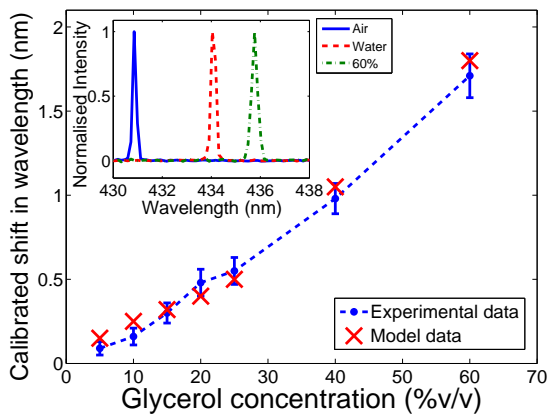

Figure 2. Main figure: comparison of experimental and theoretical results, error bars indicate standard deviation. Insert: redshift in wavelength with water and $60 \%$ glycerol.

\section{B. Sensing of polyelectrolyte adsorption}

Detection of layer-by-layer adsorption of thin films was demonstrated by adsorbing a stack of alternately-charged polyelectrolyte nano-layers to the laser surface. The polyelectrolytes used, Poly(allylamine hydrochloride) (PAH) and Poly(sodium 4-styrenesulfonate) (PSS), have been well characterized and are known to form a self-limiting monolayer 8 . Polyelectrolyte solutions $(5 \mathrm{mg} / \mathrm{mL})$ were prepared in $0.9 \mathrm{M} \mathrm{pH} 7 \mathrm{NaCl}$. Laser emission was recorded over five minutes with the laser submerged in each solution (alternating $\mathrm{PAH}$ and PSS) with a $\mathrm{NaCl}$ wash step between each change of solution. Figure 3 shows the wavelength shift relative to the shift due to $\mathrm{NaCl}$ solution for each of the polyelectrolyte layers with time. The thickness of all adsorbed layers is expected to be $\approx 80 \mathrm{~nm} 9$ demonstrating that the sensor retains its sensitivity for detection of nano-layer adsorption relatively far from the sensor surface.

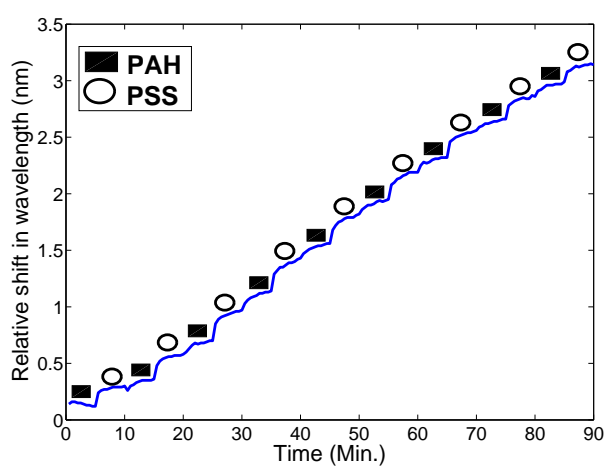

Figure 3. The increase in wavelength shift with the addition of each polyelectrolyte layer, adsorbed over 5 minutes.

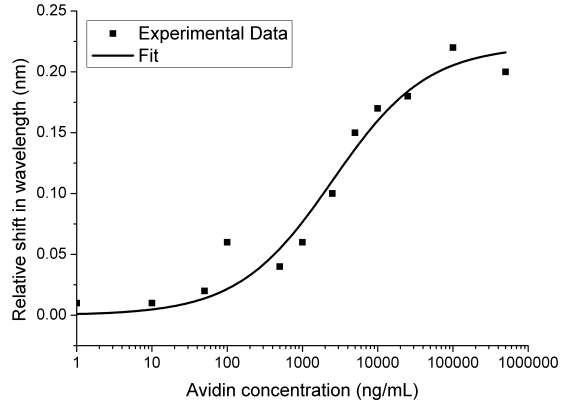

Figure 4. Specific avidin detection.

\section{Specific biomolecule sensing}

Figure 4 presents our initial results demonstrating specific detection of avidin on a biotin functionalized laser. Sensor functionalization was achieved by immersion of the sensor in polyphenylalanine lysine $(1 \mathrm{mg} / \mathrm{mL})$ prepared in $10 \mathrm{mM}$ PBS for 10 minutes before rinsing with PBS and a further submersion of the laser in NHS-LCbiotin $(0.2 \mathrm{mg} / \mathrm{mL})$ in $10 \mathrm{mM}$ PBS for 20 minutes. After rinsing (PBS), the sensor was immersed in avidin solution for 20 minutes. For each avidin concentration, a different sensor was used. The shift due to avidin adsorption on the sensor surface (i.e. the emission wavelength recorded in PBS before and after avidin exposure) is shown in figure 4. The limit of detection is $\approx 100 \mathrm{ng} / \mathrm{mL}$.

\section{Conclusion}

The sensing capabilities of an all-organic plastic DFB laser are demonstrated. Changes in refractive index of bulk solutions were measured and shown to correlate with theoretical results from a numerical model. Layerby-layer adsorption of polyelectrolytes was detected and demonstrates that the laser retains sensitivity up to $\approx 80 \mathrm{~nm}$ from the laser surface. Finally, initial results demonstrating the detection of avidin show that the limit of detection for avidin is in the region of $100 \mathrm{ng} / \mathrm{mL}$ for a biotin functionalized sensor. Future work will focus on optimisation of the laser for biomolecule detection.

\section{ACKNOWLEDGMENT}

This work was supported by EPSRC grants $\mathrm{EP} / \mathrm{J} 021962 / 1$ and EP/I029141/1.

\section{REFERENCES}

[1] M. Kristensen et al., in Optical Sensors, 2011, p. SWB1.

[2] V. Koubová et al., Sensors and Actuators B: Chemical, vol. 74, pp. $100-105,2001$.

[3] M. Baaske et al., ChemPhysChem, vol. 13, pp. 427-436, 2012.

[4] B. Cunningham et al., Sensors and Actuators B: Chemical, vol. 87 , pp. $365-370,2002$

[5] M. Lu et al., Applied Physics Letters, vol. 92, p. 261502, 2008.

[6] M. Lu et al., Applied Physics Letters, vol. 93, pp. 111113 $-111113-3$, sep 2008

[7] G. Tsiminis et al., Applied Physics Letters, 2009.

[8] J. Schmitt et al., Macromolecules, vol. 26, pp. 7058-7063, 1993.

[9] G. Ladam et al., Langmuir, vol. 16, pp. 1249-1255, 2000. 